Creat i on and char act eri zat i on of Japanese st andar ds for myocar di al perfusi on SPECT: dat abase fromt he Japanese Soci et y of $\mathrm{Nucl}$ ear Medi ci ne Wbrki ng Group

\begin{tabular}{|c|c|}
\hline 著者 & $\begin{array}{l}\text { Nakaj i ma Keni chi, Kumi ta Shi ni chi ro, I shi da } \\
\text { Yoshi o, Monose M t sur u, Hashi not o Jun, Mri i a } \\
\text { Koi chi, Taki Juni chi, Yamashi na Shohei, Mar uno } \\
\text { H r ot aka, Ogawa Nasami, Kubot a Masahi ro, } \\
\text { Takahashi Minehi ro, Odagawa Tet suro, Yokozuka } \\
\text { Koi chi }\end{array}$ \\
\hline $\begin{array}{l}\text { j our nal or } \\
\text { publ i cat i on } \mathrm{titl} \text { e }\end{array}$ & Annal s of Nucl ear Nedi ci ne \\
\hline vol une & 21 \\
\hline page $r$ ange & 505- 511 \\
\hline year & 2007-01- 01 \\
\hline URL & ht t p: //hdl . handl e. net /2297/7406 \\
\hline
\end{tabular}




\section{Creation and characterization of Japanese standards for myocardial perfusion SPECT: Database from Japanese Society of Nuclear Medicine working group activity}

Kenichi Nakajima, Shinichiro Kumita, Yoshio Ishida, Mitsuru Momose, Jun Hashimoto, Koichi Morita, Junichi Taki, Shohei Yamashina, Hirotaka Maruno, Masami Ogawa, Masahiro Kubota, Munehiro Takahashi, Tetsuro Odagawa, Koichi Yokozuka JSNM Working Group of Standardization of Myocardial SPECT in a Japanese population.

Address for correspondence:

Kenichi Nakajima, MD, Kanazawa University Hospital, 13-1 Takara-machi, Kanazawa, 920-8641, Japan;

Email:nakajima@med.kanazawa-u.ac.jp

\section{Abstract}

Objective: Standards for myocardial single-photon emission computed tomography (SPECT) adapted for a Japanese population have not been available. The purpose of this project was to create standard files approved by the Japanese Society of Nuclear Medicine and to make known the characteristics of the myocardial perfusion pattern of this population.

Methods: With the collaboration of nine hospitals, a total of 326 sets of exercise-rest myocardial perfusion images were accumulated from subjects with a low-likelihood of cardiac diseases. The normal database included a ${ }^{99 \mathrm{~m}} \mathrm{Tc}-\mathrm{MIBI} /$ tetrofosmin myocardial perfusion study with 360-degree $(\mathrm{n}=80)$ and 180-degree $(\mathrm{n}=56)$ rotations, ${ }^{201} \mathrm{Tl}$ study with 360 -degree $(\mathrm{n}=115)$ and 180-degree rotations $(\mathrm{n}=54)$ and a dual-isotope study with 360 -degree rotation $(\mathrm{n}=27)$. The projection images were transferred by DICOM (Digital Imaging and Communications in Medicine) format and reconstructed and analyzed with polar maps.

Results: The projection data from multiple centers were successfully transferred to a common format for SPECT reconstruction. When the average values were analyzed using a 17-segment model, myocardial counts in the septal segment differed significantly between 180-degree and 360-degree rotation acquisitions. Regional differences were observed between males and females in the inferior and anterior regions. A tracer difference between ${ }^{99 \mathrm{~m}} \mathrm{Tc}$ and ${ }^{201} \mathrm{Tl}$ was also observed in some segments. The attenuation patterns differed significantly between subjects from the United States and Japanese populations.

Conclusion: Myocardial perfusion data that were specific for the Japanese population were generated. The normal database can serve a standard for nuclear cardiology work conducted in Japan.

Keywords:

Myocardial perfusion imaging, SPECT, Standard, Japanese population, Working Group of the Japanese Society of Nuclear Medicine

\section{Introduction}

Myocardial SPECT has been utilized as an important tool for optimal decision making in cardiology. The Guidelines for the Clinical Use of Cardiac Radionuclide Imaging were originally published in 1986 in the USA and updated in 1995. ${ }^{1}$ In Japan, Nuclear Cardiology Guidelines were also summarized and published in 2005 by the task force of the Japan Circulation Society, Japanese Society of Nuclear Medicine (JSNM) and related societies, and the role of nuclear modalities has been recognized. ${ }^{2}$ Among non-invasive technologies currently being developed in the fields of echocardiography, multi-detector $\mathrm{x}$-ray computed tomography and magnetic resonance imaging, nuclear medicine plays a pivotal role in evaluating myocardial perfusion and metabolism.

To be accepted as a reliable technology, standard patterns of myocardial radiotracer distribution and function should be defined. However, because myocardial distribution is known to be affected by factors of data acquisition methods, radiopharmaceutical types and gender, one of the most important issues is the standardization of myocardial SPECT studies. Moreover, since the differences in body habitus are not negligible, standards cannot be used interchangeably among populations.

In light of this background, the Japanese Society of Nuclear Medicine started working on a group project for creating myocardial standard based on a SPECT study from 2006 during a two-year period. The goal of the project was to create normal database and standard files for computer software and outline characteristics of a Japanese population with respect to perfusion, fatty acid and sympathetic imaging. This first report presents and provides data on the characteristics of a Japanese population with respect to myocardial perfusion SPECT. 


\section{Methods}

\section{Participating institutions}

The members of the working group and those who cooperated with the accumulation and analysis of the SPECT data are listed in Table 1. Nine hospitals collaborated on the accumulation of the myocardial SPECT images. Five venders of SPECT and two radiopharmaceutical companies were also involved. We also consulted the developers of the major myocardial SPECT quantification software, namely, Quantitative Gated SPECT and Perfusion SPECT (QGS/QPS, Cedars-Sinai Medical Center, Los Angeles, CA), the Emory Cardiac Toolbox (ECTb; Emory University, Atlanta, GA) and 4D-MSPECT (University of Michigan Medical Center, Ann Arbor, MI). ${ }^{3-10}$ The data collection was approved in each hospital by the appropriate ethical committee or review board.

\section{Definition of study population}

Selection criteria of subjects with a low likelihood of cardiac disease are as follows. Regarding stress type, only subjects who had taken part in exercise were included, while subjects who underwent pharmacological stress were not. The subjects had no electrocardiographic abnormalities indicative of ischemia and no underlying cardiac diseases. Subjects with hypertension and diabetes mellitus that required medication were excluded. Subjects with inappropriate arrhythmia for electrocardiography (ECG)-gated SPECT data collection were excluded. Gated SPECT was required for ${ }^{99 \mathrm{~m} T c-}$ hexakis(2-methoxy-isobutyl isonitrile) (MIBI) and tetrofosmin and if possible for ${ }^{201} \mathrm{Tl}$. The data of MIBI and tetrofosmin were not separated. Wall motion determined by gated SPECT should have been normokinetic. Both patients who had normal coronary arteriography and those who were not indicated for coronary arteriography because of a low possibility of ischemic heart disease were included. The standard administration dose of ${ }^{99 \mathrm{~m}} \mathrm{Tc}$ perfusion tracers and ${ }^{201} \mathrm{Tl}$ depended on the institutions. However, the tracer dose and SPECT acquisition parameters were confirmed in each institute, and were judged appropriate for reliable results. Either low-energy high-resolution (LEHR) or general-purpose (LEGP) collimators were used. A projection set of 360-degree rotation derived from dual- $(\mathrm{n}=17)$ or triple-detector $(\mathrm{n}=205)$ cameras, and a projection set of 180-degree rotation from dual- $(\mathrm{n}=90)$ or triple- detector $(\mathrm{n}=14)$ cameras with rectangular or 76-degree rotation range were used. A circular orbit was used in 153 patients and a non-circular orbit in 173 patients. The acquisition angle for each projection was 4-6 degrees per step. No attenuation correction method was used in any of the hospitals. For gated acquisition, division of RR interval was $16(n=79), 10(n=49)$ and $8(n=8)$ frames for ${ }^{99 \mathrm{~m} T c}$ tracers, while it was $16(\mathrm{n}=100)$ and $8(n=69)$ frames for ${ }^{201} \mathrm{Tl}$ study.

\section{Data transfer and reconstruction}

The data included exercise and rest imaging for ${ }^{99 \mathrm{~m}} \mathrm{Tc}$ tracers, and exercise and delayed imaging for ${ }^{201} \mathrm{Tl}$, in which ${ }^{201} \mathrm{Tl}$ reinjection studies (17 of 169 patients, 10\%) were also included. The SPECT projection data with gated and ungated (or sum of the gated images) were anonymously transferred by the original or DICOM formats. All the data were accumulated at Kanazawa University. The SPECT data format was modified, if necessary, to the appropriate format for further processing. The SPECT reconstruction was performed with a Butterworth prefilter and a ramp filter using the same reconstruction parameters. The cut-off frequency of the Butterworth filter was 0.45 and 0.40 cycles $/ \mathrm{cm}$ for gated and ungated acquisition, respectively. The same software (Xeleris, GE Yokogawa Medical Systems, Ltd.) was used for generating gated and ungated shortaxis images.

\section{Polar map and segment-based analysis}

The QGS/QPS, ECTb and 4D-MSPECT software were available to us for results. ${ }^{3-10}$ In this manuscript, a QPS software algorithm was used to generate perfusion polar maps and to characterize the mean and the mean deviation of 17 segments. The QPS algorithm used the normalization of the maximal values so that normal segments had $100 \%$ values to eliminate errors due to maxima located in small hot spots. ${ }^{11,12}$ Gated SPECT quantification was used to confirm normal ejection fraction, wall motion and volumes using the QGS software.

\section{Statistics}

The parameters were expressed as the average and standard deviation. For the polar map quantification, the average and mean deviations were calculated based on the database editor of the QPS software. The differences in segmental values were calculated based on unpaired $\mathrm{T}$ test after converting the mean deviation to standard deviation assuming normal distribution. A p value of less than 0.05 was considered significant. $\mathrm{p}$ values between 0.05 and 0.1 were considered borderline significant. 


\section{Background of subjects}

The patient background regarding age, height, weight, heart rate, blood pressure, ejection fraction and left ventricular volumes is summarized in Table 2 . Left ventricular volumes divided by body surface area are shown as volume index $(\mathrm{mL} /$ $\mathrm{m}^{2}$ ). For ${ }^{201} \mathrm{Tl}$ SPECT studies, all 180-degree data were acquired by ECG gating, and no significant difference was observed between tracers in terms of age, stature, heart rate or blood pressure. When EFs for the 180 -degree data from ${ }^{201} \mathrm{Tl}$ and ${ }^{99 \mathrm{~m}} \mathrm{Tc}$ studies were compared, the ${ }^{201} \mathrm{Tl}$ study showed lower EF than the 99m Tc study in females $(p=0.016)$ and males $(p=0.038)$. However, the ${ }^{201} \mathrm{Tl} 360$-degree data were ungated and precise information for ventricular function was not available.

\section{Statistical differences in polar map analysis}

Average segmental values were calculated based on 17segment models for post-stress and resting conditions. The polar maps were compared as shown in Figure 1. Table 3 shows the average values of mid and apical segments for females and males with ${ }^{99 \mathrm{~m} T c}$ and ${ }^{201} \mathrm{Tl}$ tracers in resting condition. Since the segment of the normal regions was adjusted to $100 \%$ in the QPS algorithm, values greater than $100 \%$ were included.

When the 360-degree and 180-degree rotation methods were compared, septal segments showed higher values in the 180-degree rotation for both ${ }^{99 \mathrm{~m} T c}$ and ${ }^{201} \mathrm{Tl}$. Gender differences were significant in the mid and apical inferior segments, showing lower values in males. Mid anterior segments showed significant differences between the 360 and 180 -degree rotations for ${ }^{99} \mathrm{mTc}$ but not for ${ }^{201} \mathrm{Tl}$. Apical anterior segments showed gender differences, namely, values were lower in females in ${ }^{201} \mathrm{Tl}$ but not

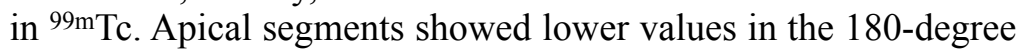
rotation than in the 360-degree rotation. Differences in radionuclide were observed regarding the segments of statistical significance in the mid anterior segment and the apex.

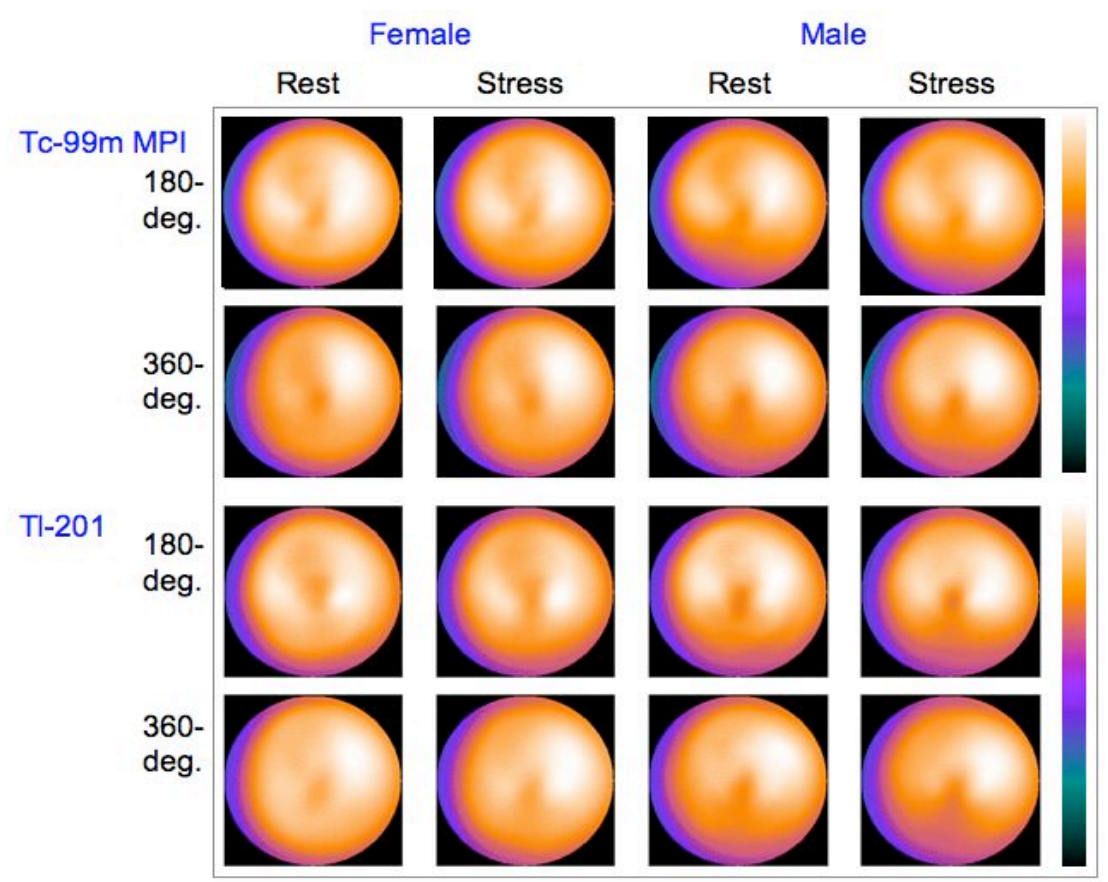

Table 1. Working group members and participating institutes for JSNM myocardial standards

\section{Chief}

Kanazawa University Hospital: Kenichi

Nakajima*

Medical Institutes

Nippon Medical School Hospital:

Shinichiro Kumita*, Yoshimitsu Fukushima

National Cardiovascular Center: Yoshio

Ishida*

Keio University Hospital: Jun Hashimoto*

Tokyo Women's Medical University:

Mitsuru Momose*

Hokkaido University Hospital: Koichi

Morita*, Masayuki Inubushi, Keiichiro

Yoshinaga

Toho University Omori Medical Center:

Shohei Yamashina*, Junichi Yamazaki

Toranomon Hospital: Hirotaka Maruno*

Kanazawa University Hospital: Koichi

Okuda, Tatsuya Yoneyama, Nasima Akhter,

Junichi Taki*

Kanazawa Cardiovascular Center: Masaya

Kawano

Manufacturers of SPECT systems

GE Yokogawa Medical Systems: Masami Ogawa*

Toshiba Medical Systems Corporation:

Masahiro Kubota*, Nobutoku Motomura

Siemens-Asahi Medical Technologies:

Tetsuro Odagawa*

Hitachi Medical Corporation: Koichi

Yokotsuka*

Shimadzu Corporation: Munehiro

Takahashi*, Yuichi Inaoka

Radiopharmaceutical Companies

FUJIFILM RI Pharma: Takehiro Ishikawa,

Katsunori Yoshioka

Nihon Medi-Physics: Junichi Kato,

Hideharu Niida

Acknowledgement for creating standards

Cedars-Sinai Medical Center: Guido

Germano, Piotr Slomka, Geoff Pollard

Emory University Hospital: Ernest Garcia

University of Michigan Medical Center: Edward Ficaro

* Working group members

\section{Figure 1}

Standard polar maps of the average values for

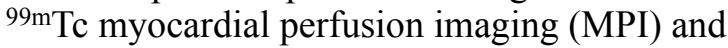
${ }^{201} \mathrm{Tl}$. Note the difference in the septal count between 180-degree and 360-degree acquisition protocols. Gender differences in the anterior and inferior regions are also demonstrated. 
Table 2. Backgound of patients with myocardial perfusion gated SPECT

\begin{tabular}{|c|c|c|c|c|c|c|}
\hline \multirow{3}{*}{$\begin{array}{l}\text { Tracer } \\
\text { Camera rotation } \\
\text { Gender }\end{array}$} & \multicolumn{4}{|c|}{ Tc-99m MIBI and tetrofosmin } & \\
\hline & \multicolumn{2}{|l|}{ 180-deg. } & \multicolumn{2}{|l|}{ 360-deg. } & & 180-deg. \\
\hline & Female & Male & Female & Male & Female & Male \\
\hline No. of patients & 28 & 28 & 40 & 40 & 27 & 27 \\
\hline Age & $61 \pm 10$ & $57 \pm 15$ & $59 \pm 16$ & $63 \pm 12$ & $64 \pm 10$ & $66 \pm 9$ \\
\hline Height $(\mathrm{cm})$ & $155 \pm 7$ & $168 \pm 6$ & $155 \pm 6$ & $164 \pm 6$ & $155 \pm 6$ & $165 \pm 7$ \\
\hline Weight (kg) & $60 \pm 8$ & $68 \pm 12$ & $51 \pm 7$ & $63 \pm 9$ & $56 \pm 8$ & $64 \pm 8$ \\
\hline $\operatorname{BSA}\left(\mathrm{m}^{2}\right)$ & $1.55 \pm 0.12$ & $1.71 \pm 0.16$ & $61.44 \pm 0.12$ & $1.64 \pm 0.14$ & $1.50 \pm 0.09$ & $1.66 \pm 0.12$ \\
\hline \multicolumn{7}{|l|}{ HR and BP at rest } \\
\hline $\mathrm{HR}(/ \min )$ & $75 \pm 13$ & $67 \pm 12$ & $63 \pm 10$ & $67 \pm 11$ & $74 \pm 12$ & $71 \pm 12$ \\
\hline $\mathrm{SP}(\mathrm{mmHg})$ & $145 \pm 30$ & $135 \pm 16$ & $125 \pm 15$ & $133 \pm 14$ & $152 \pm 23$ & $138 \pm 16$ \\
\hline $\mathrm{DP}(\mathrm{mmHg})$ & $82 \pm 11$ & $84 \pm 15$ & $71 \pm 10$ & $79 \pm 10$ & $86 \pm 15$ & $84 \pm 14$ \\
\hline \multicolumn{7}{|c|}{$H R$ and $B P$ at peak exercise } \\
\hline $\mathrm{HR}(/ \min )$ & $129 \pm 20$ & $122 \pm 21$ & $129 \pm 23$ & $132 \pm 18$ & $131 \pm 22$ & $129 \pm 15$ \\
\hline $\mathrm{SP}(\mathrm{mmHg})$ & $188 \pm 39$ & $203 \pm 33$ & $192 \pm 25$ & $205 \pm 25$ & $206 \pm 22$ & $220 \pm 26$ \\
\hline $\mathrm{DP}(\mathrm{mmHg})$ & $90 \pm 17$ & $96 \pm 34$ & $97 \pm 23$ & $98 \pm 23$ & $102 \pm 21$ & $113 \pm 23$ \\
\hline \multicolumn{7}{|l|}{ Rest condition } \\
\hline EF $(\%)$ & $71 \pm 8$ & $65 \pm 6$ & $68 \pm 7$ & $63 \pm 7$ & $65 \pm 8$ & $62 \pm 5$ \\
\hline EDV (mL) & $70 \pm 14$ & $89 \pm 16$ & $60 \pm 10$ & $76 \pm 14$ & $62 \pm 11$ & $73 \pm 15$ \\
\hline ESV (mL) & $21 \pm 7$ & $31 \pm 9$ & $20 \pm 6$ & $28 \pm 8$ & $22 \pm 7$ & $28 \pm 8$ \\
\hline $\operatorname{EDVI}\left(\mathrm{mL} / \mathrm{m}^{2}\right)$ & $45 \pm 8$ & $52 \pm 8$ & $41 \pm 6$ & $47 \pm 9$ & $41 \pm 8$ & $44 \pm 9$ \\
\hline $\operatorname{ESVI}\left(\mathrm{mL} / \mathrm{m}^{2}\right)$ & $14 \pm 5$ & $19 \pm 5$ & $13 \pm 4$ & $17 \pm 5$ & $15 \pm 5$ & $17 \pm 5$ \\
\hline \multicolumn{7}{|c|}{ Post-stress condition } \\
\hline $\mathrm{EF}(\%)$ & $71 \pm 8$ & $66 \pm 7$ & $69 \pm 6$ & $63 \pm 7$ & $67 \pm 7$ & $64 \pm 8$ \\
\hline EDV (mL) & $69 \pm 15$ & $82 \pm 15$ & $59 \pm 10$ & $72 \pm 14$ & $59 \pm 13$ & $69 \pm 14$ \\
\hline ESV (mL) & $21 \pm 8$ & $28 \pm 8$ & $19 \pm 6$ & $27 \pm 7$ & $20 \pm 6$ & $25 \pm 8$ \\
\hline $\operatorname{EDVI}\left(\mathrm{mL} / \mathrm{m}^{2}\right)$ & $44 \pm 8$ & $48 \pm 7$ & $40 \pm 6$ & $44 \pm 9$ & $40 \pm 8$ & $42 \pm 8$ \\
\hline $\operatorname{ESVI}\left(\mathrm{mL} / \mathrm{m}^{2}\right)$ & $14 \pm 5$ & $16 \pm 4$ & $13 \pm 4$ & $16 \pm 5$ & $13 \pm 4$ & $15 \pm 5$ \\
\hline
\end{tabular}

$\mathrm{HR}$, heart rate; SP, systolic pressure; DP, diastolic pressure

EF, ejection fraction; EDV, end-diastolic volume; ESV, end-systolic volume 
Table 3. Segmental average values in the polar map at resting condition

\begin{tabular}{|c|c|c|c|c|c|c|c|c|}
\hline \multirow{3}{*}{ Sgments } & \multicolumn{4}{|c|}{ Average values } & \multicolumn{4}{|l|}{$\mathrm{P}$ values } \\
\hline & \multirow{2}{*}{$\begin{array}{l}180 \text { deg. } \\
\text { Female }\end{array}$} & \multirow{2}{*}{$\begin{array}{l}360 \text { deg. } \\
\text { Female }\end{array}$} & \multirow{2}{*}{$\begin{array}{r}180 \\
\text { deg. } \\
\text { Male }\end{array}$} & \multirow{2}{*}{$\begin{array}{l}360 \\
\text { deg. } \\
\text { Male }\end{array}$} & \multicolumn{2}{|c|}{180 vs $360 \mathrm{deg}$. } & \multicolumn{2}{|c|}{ Female vs Male } \\
\hline & & & & & Female & Male & $\begin{array}{l}180 \\
\text { deg. }\end{array}$ & $360 \mathrm{deg}$. \\
\hline \multicolumn{9}{|c|}{ Tc-99m MIBI/Tetrofosmin } \\
\hline Mid A & 92 & 96 & 92 & 96 & $\begin{array}{ll}6 & 0.008\end{array}$ & 0.007 & ns & ns \\
\hline Mid AS & 92 & 87 & 94 & 87 & $7 \quad 0.0003$ & $<0.0001$ & ns & ns \\
\hline Mid IS & 89 & 85 & 84 & 82 & $2 \quad 0.009$ & ns & 0.002 & 0.024 \\
\hline Mid I & 89 & 91 & 81 & 84 & ns & 0.024 & $<0.0001$ & $<0.0001$ \\
\hline Mid IL & 99 & 99 & 96 & 97 & ns & ns & ns $(0.06)$ & ns \\
\hline Mid AL & 104 & 108 & 106 & 108 & $8 \quad 0.016$ & ns & ns & ns \\
\hline Apical A & 93 & 97 & 96 & 100 & 0.025 & 0.010 & $\mathrm{~ns}(0.078)$ & ns $(0.05)$ \\
\hline Apical S & 99 & 95 & 98 & 95 & $5 \quad 0.016$ & ns $(0.0822)$ & ns & ns \\
\hline Apical I & 92 & 92 & 88 & 87 & ns & ns & 0.009 & 0.0004 \\
\hline Apical L & 104 & 105 & 106 & 105 & ns & $\mathrm{ns}$ & ns & ns \\
\hline Apex & 92 & 91 & 93 & 92 & ns & ns & ns & ns \\
\hline \multicolumn{9}{|l|}{ Tl-201 } \\
\hline Mid A & 94 & 94 & 97 & 96 & ns & ns & ns & ns $(0.10)$ \\
\hline Mid AS & 95 & 90 & 96 & 89 & $\begin{array}{ll}9 & 0.002\end{array}$ & $<0.0001$ & ns & ns \\
\hline Mid IS & 90 & 91 & 86 & 84 & ns & ns & 0.039 & $<0.0001$ \\
\hline Mid I & 90 & 92 & 82 & 83 & ns & ns & $<0.0001$ & $<0.0001$ \\
\hline Mid IL & 97 & 97 & 94 & 95 & ns & ns & ns & ns \\
\hline Mid AL & 101 & 105 & 104 & 107 & $\begin{array}{ll}7 & 0.028\end{array}$ & ns $(0.07)$ & ns & ns \\
\hline Apical A & 93 & 97 & 98 & 100 & 0.021 & ns & 0.020 & 0.012 \\
\hline Apical S & 100 & 97 & 100 & 95 & $5 \mathrm{~ns}(0.07)$ & 0.0034 & ns & $\mathrm{ns}(0.10)$ \\
\hline Apical I & 95 & 93 & 87 & 87 & ns & ns & 0.0002 & $<0.0001$ \\
\hline Apical L & 102 & 100 & 103 & 102 & ns & ns & ns & ns \\
\hline Apex & 90 & 93 & 88 & 92 & $2 \quad 0.043$ & 0.010 & ns & ns \\
\hline
\end{tabular}

Abbreviations

A, anterior; AS, anteroseptal; IS, inferoseptal; I, inferior; IL, inferolateral; AL, anterolateral; deg., degrees; ns, not significant 


\section{Discussion}

As a working group activity of the Japanese Society of Nuclear Medicine from 2006 to 2007, standards for myocardial perfusion SPECT were generated. The major conclusion of this study is that normal patterns were specific for genders, rotation angles and radionuclide used in the study. The importance of population specific standards should be emphasized. Normal perfusion files can serve as standards for clinical examinations as well as for multicenter studies.

\section{Necessity of creating standards as working group} activity

Quantification of myocardial perfusion as well as ventricular function is considered important for the detection of myocardial ischemia and defect size. These standards created from a normal database can also serve as appropriate guides for treatment and predicting prognosis of patients after acute and chronic ischemic syndromes. ${ }^{1,2}$ Although standard perfusion patterns have been determined by some institutions and used for clinical practice, they may not be readily applicable to users' own institutions. Application of standards made for the US population to a Japanese population may also create inaccuracies in quantification. However, no authorized myocardial standards have been established by Nuclear Medicine Societies. Therefore, this working group was started to create common standard files that would be fitted to a Japanese population.

\section{Need for specific population and technology}

Appropriate standards that are specific to the population should be used. The precision of gated SPECT results were examined in the current multicenter study and found to be excellent. ${ }^{13}$ Nearnormal patients were selected and analyzed from a JACCESS study (Japanese investigation for prognosis based on gated SPECT, Tsunehiko Nishimura as the chief investigator). ${ }^{14} \mathrm{~A}$ total of 268 patients were selected from candidates with no baseline cardiac diseases and with no cardiac events over a three-year period. Ejection fractions for women and men were
$74 \% \pm 9 \%$ and $63 \% \pm 7 \%(\mathrm{p}<0.0001)$. The present working group study also showed higher EF in females than in males by 5-6\%. Left ventricular volumes were significantly smaller in women than in men. Important factors for the studies in a Japanese population included a high incidence of small hearts in women and the relatively advanced age of the population. As for the regional perfusion patterns, similar differences due to differences in body habitus and ethnicity may be anticipated. The major SPECT technology and preference may also differ among countries. Approximately half of SPECT acquisition is performed with 360-degree rotation in Japan, while 180-degree rotation is more commonly used in the US. Some institutions prefer ${ }^{201} \mathrm{Tl}$ studies to ${ }^{99 \mathrm{~m}} \mathrm{Tc}$ imaging agents. Considering the diversity of preferences in myocardial perfusion studies, individual data catering to the need in this country should be provided.

\section{Benefits and limitations based on multicenter data}

The standards established by the working group contained various acquisition conditions with standard protocols but with minor differences. The standards provided an average pattern from several institutions, which have benefits as well as drawbacks. Strictly speaking, the database generated by each of the institutions would be more specific when considering the differences in technology, study protocol and targets of each study. However, accumulation of nearnormal subjects based on strict criteria may be difficult for all institutions. The major merit of using authorized standards is that they can be utilized safely in most of the institutions where standard SPECT protocols are used. A common database can also be used for multicenter studies including nuclear cardiology techniques. However, as shown in this study, differences in rotation angle range, gender and tracers are important determinants affecting standard patterns. The factors ignored in this standard included differences in LEHR and LEGP collimators, differences in ${ }^{99 \mathrm{~m}} \mathrm{Tc}$ MIBI and tetrofosmin, and differences in noncircular and noncircular orbit. The last factor might be re-considered when a sufficient number of data have been accumulated. The dual nuclide study may be an option for some hospitals and the database could be of additional benefit.

\begin{abstract}
Figure 2
Standard polar map patterns made by US population (Cedars Sinai Medical Center, CA, USA) with 40 males and 40 females ${ }^{11}$. The maps were obtained by 180-degree rotation in the supine position. The maps from a Japanese population are shown for comparison (right).
\end{abstract}

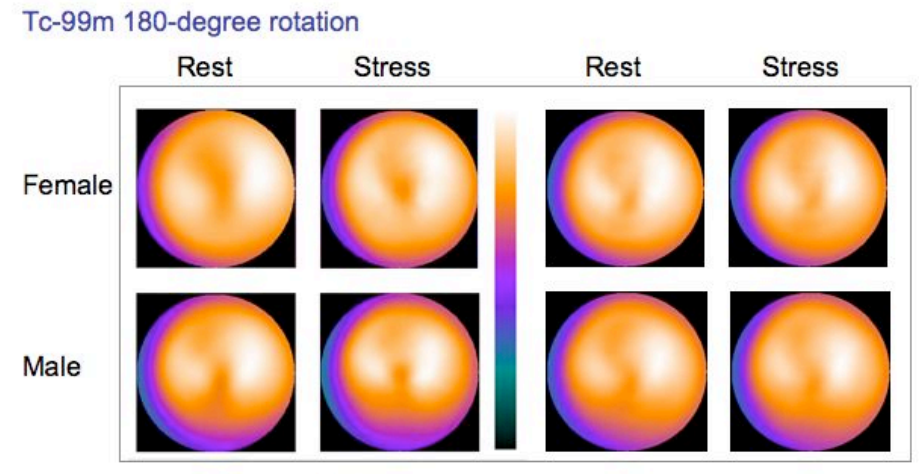

US population 
Differences of standards between the United States and Japan

When the normal databases from the US and Japan were compared, some differences should be noted. Figure 2 shows an example of maps based on US and Japanese standards. In the comparison, attenuation in the anterior wall seemed to be greater in females in the US population than in the Japanese population (93-85\% vs. $93-92 \%$ in the apical anterior segment to the apex). ${ }^{11}$ The inferior wall also showed lower values in males in the US population than in the Japanese population $(76 \%$ vs. $81 \%$ in the mid inferior segment). The gender difference was considered to be larger in the US population than in the Japanese population. The differences seem to reflect the difference in attenuation from female breasts and male diaphragm. In addition, the difference in the same gender could not be explained by body weight alone. Thus, transferring application of the US standards to a Japanese population is considered inappropriate.

\section{Future direction}

The standards are prepared to offer the normal database and computer files to nuclear medicine institutions. Their validity and diagnostic accuracy should be examined in subsequent studies. The parameters derived from a gated study could be calculated for standardization using these compiled studies. Considering the current use of an attenuation correction method, new sets of standards may be required in the near future. Additional important fields requiring a standard database are ${ }^{123}$ I-BMIPP and ${ }^{123} \mathrm{I}-$ MIBG. SPECT data accumulation for ${ }^{123} I$ tracers is in progress and will be completed soon.

\section{Conclusion}

Myocardial perfusion data specific for the Japanese population were generated by the JSNM working group for standardization of myocardial SPECT. Significant segmental differences were observed based on the SPECT rotation angles, gender and tracers. The normal database can serve as a standard for common myocardial stress protocols and for multi-center comparative studies involving myocardial SPECT.

\section{Acknowledgment}

We thank the many physicians and technologists who contributed to the accumulation and generation of the normal database. We also thank GE Yokogawa Medical Systems for providing a workstation for data processing. The research version of QPS software was kindly supplied by Drs. Germano and Slomka (Cedars Sinai Medical Center). This work was performed as part of the activity of the JSNM working group activity and supported partly by Grants-in-Aid for Scientific Research in Japan. 


\section{References}

1. Klocke FJ, Baird MG, Lorell BH, Bateman TM, Messer JV, Berman DS, et al. ACC/ AHA/ASNC guidelines for the clinical use of cardiac radionuclide imaging--executive summary: a report of the American College of Cardiology/American Heart Association Task Force on Practice Guidelines (ACC/ AHA/ASNC Committee to Revise the 1995 Guidelines for the Clinical Use of Cardiac Radionuclide Imaging). Circulation 2003;108:1404-18.

2. Tamaki N. Guidelines for Clinical Use of Cardiac Nuclear Medicine (JCS 2005). Circulation J 2005;69 Suppl. IV:1125-202.

3. Faber TL, Cooke CD, Folks RD, Vansant JP, Nichols KJ, DePuey EG, et al. Left ventricular function and perfusion from gated SPECT perfusion images: an integrated method. $J$ Nucl Med 1999;40:650-9.

4. Faber TL, Santana CA, Garcia EV, CandellRiera J, Folks RD, Peifer JW, et al. Threedimensional fusion of coronary arteries with myocardial perfusion distributions: clinical validation. J Nucl Med 2004;45:745-53.

5. Germano G, Berman DS. Gated myocardial perfusion SPECT. In: Dilsizian V, Narula J, eds. Atlas of nuclear cardiology. Philadelphia: Current Medicine, Inc., 2003:167-82

6. Germano G, Kiat H, Kavanagh PB, Moriel M, Mazzanti M, Su HT, et al. Automatic quantification of ejection fraction from gated myocardial perfusion SPECT. $J$ Nucl Med 1995;36:2138-47.

7. Lipke CS, Kuhl HP, Nowak B, Kaiser HJ, Reinartz P, Koch KC, et al. Validation of 4DMSPECT and QGS for quantification of left ventricular volumes and ejection fraction from gated 99mTc-MIBI SPET: comparison with cardiac magnetic resonance imaging. Eur $J$ Nucl Med Mol Imaging 2004;31:482-90.

8. Lum DP, Coel MN. Comparison of automatic quantification software for the measurement of ventricular volume and ejection fraction in gated myocardial perfusion SPECT. Nucl Med Commun 2003;24:259-66.

9. Nakajima K, Higuchi T, Taki J, Kawano M, Tonami N. Accuracy of ventricular volume and ejection fraction measured by gated myocardial SPECT: comparison of 4 software programs. $J$ Nucl Med 2001;42:1571-8.

10. Nichols K, Santana CA, Folks R, Krawczynska E, Cooke CD, Faber TL, et al.
Comparison between ECTb and QGS for assessment of left ventricular function from gated myocardial perfusion SPECT. $J$ Nucl Cardiol 2002;9:285-93.

11. Nishina H, Slomka PJ, Abidov A, Yoda S, Akincioglu C, Kang X, et al. Combined supine and prone quantitative myocardial perfusion SPECT: method development and clinical validation in patients with no known coronary artery disease. $J \mathrm{Nucl} \mathrm{Med}$ 2006;47:51-8.

12. Slomka PJ, Nishina H, Berman DS, Kang X, Friedman JD, Hayes SW, et al. Automatic quantification of myocardial perfusion stress-rest change: a new measure of ischemia. $J$ Nucl Med 2004;45:183-91.

13. Nakajima K, Nishimura T. Inter-institution preference-based variability of ejection fraction and volumes using quantitative gated SPECT with $(99 \mathrm{~m})$ Tc-tetrofosmin: a multicentre study involving 106 hospitals. Eur $J$ Nucl Med Mol Imaging 2006;33:127-33.

14. Nakajima K, Kusuoka $H$, Nishimura S, Yamashina A, Nishimura T. Normal limits of ejection fraction and volumes determined by gated SPECT in clinically normal patients without cardiac events: a study based on the J-ACCESS database. Eur J Nucl Med Mol Imaging 2007;34:1088-96. 